\title{
Hyponatraemia secondary to an inappropriately high release of antidiuretic hormone in cardiac tamponade
}

\author{
Peter H Groves, Ajay M Shah, Stephen J Hutchison
}

\begin{abstract}
A spontaneous intrapericardial haemorrhage caused cardiac tamponade in a 29 year old paraplegic man who was being treated with warfarin. The associated persistent hyponatraemia, which was believed to be caused by an inappropriately high release of antidiuretic hormone, rapidly resolved after pericardiocentesis.
\end{abstract}

Release of antidiuretic hormone increases when cardiac tamponade develops. ${ }^{1}$ The concentration of serum sodium is usually normal because the balance between fluid and sodium is maintained. We report a case of cardiac tamponade in which excessive fluid retention caused by an inappropriately high release of antidiuretic hormone led to prolonged hyponatraemia.

\section{Case report}

A 29 year old man was transferred to the regional cardiothoracic centre from a local spinal rehabilitation unit, to which he had been admitted five months before with traumatic tetraplegia at the level of the sixth cervical vertebrae. The day after his admission to the spinal unit he was treated with warfarin because of symptoms and signs suggestive of pulmonary embolism. Maintenance treatment with warfarin was continued and he also received dantrolene, baclofen, lactulose, and intermittent courses of cephradine and gentamicin for urinary infections. Over three weeks he became progressively more short of breath, but examination on several occasions showed no diagnostic signs referable to his heart or chest. Initial blood tests showed a fall in haemoglobin concentration to $73 \mathrm{~g} / 1$ and serum sodium to $117 \mathrm{mmol} / \mathrm{l}$ and a rise in the serum concentration of aspartate transaminase to $102 \mathrm{IU} / 1$ (normal range 12-30). All other tests of electrolytes and liver function were within the normal range.

Though the dose of warfarin was not changed his International Normalised Ratio rose to 10 the day before his transfer; previously it had been well controlled. A chest $x$ ray showed considerable cardiomegaly with pulmonary congestion. On admission to the cardiology unit the slightest exertion made him breathless; he did not have a fever, his heart rate was 90 beats per minute, and his blood pressure was $95 / 70 \mathrm{~mm} \mathrm{Hg}$ with $10 \mathrm{~mm} \mathrm{Hg}$ of pulsus paradoxus. Jugular venous pressure was considerably elevated and heart sounds were quiet with no added sounds. An electrocardiogram showed sinus rhythm with low voltage complexes and $T$ wave inversion in the inferior leads. Cross sectional echocardiography confirmed the presence of a large pericardial effusion with diastolic collapse of both the right atrium and right ventricle which with the physical signs confirmed the diagnosis of cardiac tamponade. Treatment with warfarin was stopped and its effects were reversed with fresh frozen plasma and vitamin $\mathrm{K}$. The pericardial effusion was tapped, yielding $1500 \mathrm{ml}$ of heavily bloodstained fluid; a pigtail catheter was left in the pericardial cavity as a drain. The patient had considerable and almost immediate symptomatic relief. A further $500 \mathrm{ml}$ of bloodstained fluid drained over the next 48 hours by which time only a small amount of residual pericardial fluid was detectable by echocardiography. The fluid contained protein $(56 \mathrm{~g} / \mathrm{l})$; cytology did not show any malignant cells and all
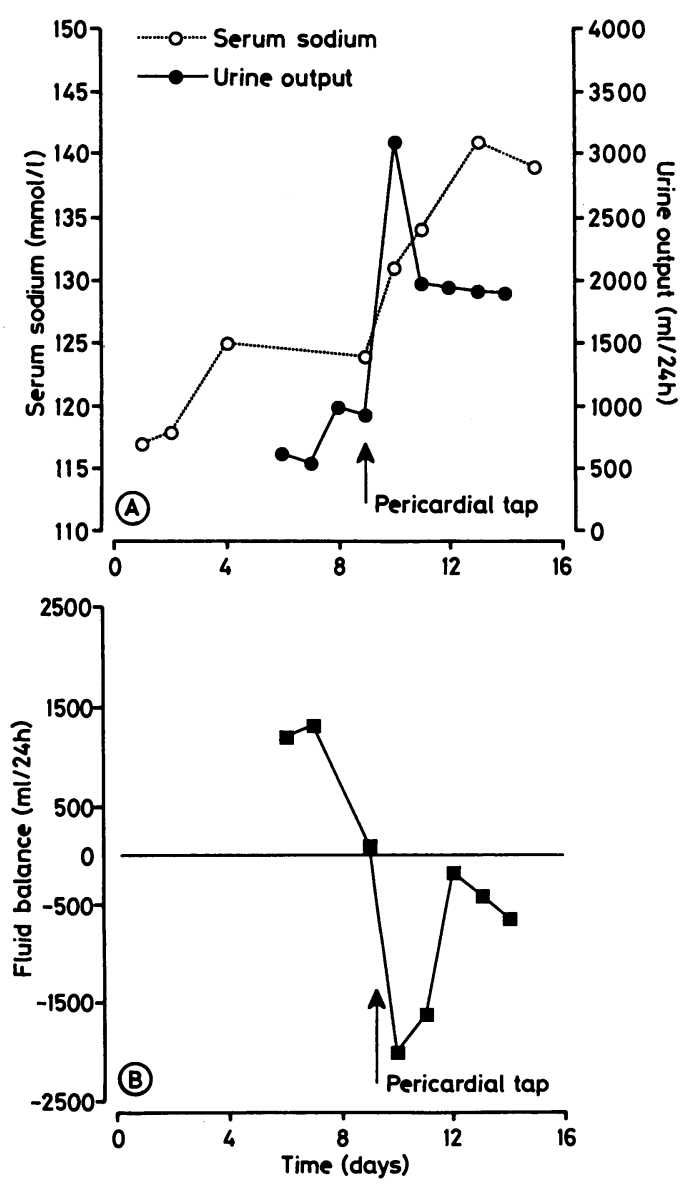

Changes in $(A)$ serum sodium and urine output and $(B)$ fluid balance before and after pericardial tap. 
microbiological tests were negative. Immediately after the pericardial fluid was removed the patient had a considerable diuresis and the serum sodium returned to normal within 36 hours without further treatment (figure). At repeat echocardiography one week and four weeks later there was no reaccumulation of pericardial fluid.

\section{Discussion}

Hyponatraemia caused by an inappropriately high release of antidiuretic hormone has not previously been reported in cardiac tamponade. The haemodynamic effects of cardiac tamponade acting via arterial and atrial baroreceptors are a potent stimulus to the release of antidiuretic hormone. ${ }^{1}$ This response, combined with raised serum concentrations of catecholamines and activation of the renin angiotensin system, is aimed at maintaining arterial blood pressure in the face of a falling cardiac output. ${ }^{2}$ The release of atrial natriuretic factor is suppressed (because atrial stretch is reduced ${ }^{34}$ ) and an increase in renal sympathetic outflow increases tubular reabsorption of sodium. ${ }^{5}$ The overall physiological response to cardiac tamponade is therefore away from natriuresis and diuresis and the balance of these responses is such that a normal serum sodium is usually maintained. Our patient had prolonged and severe hyponatraemia when progressive cardiac tamponade developed after spontaneous intrapericardial haemorrhage. Apart from a short period of treatment with intravenous fluids in the early stages of this illness, we found no other factor that could have exacerbated or caused the hyponatraemia. Furthermore, the rapid return to normal of serum sodium after pericardiocentesis led us to conclude that the hyponatraemia and cardiac tamponade were directly related. The figure shows that the rise in serum sodium after the removal of the pericardial fluid was paralleled by an increase in urine output. This close relation suggests that the hyponatraemia was caused by intravascular volume expansion and that its rapid resolution resulted from the diuresis that followed pericardial drainage. Though fluid retention would normally be expected in the presence of cardiac tamponade, the extent of the volume changes that led to hyponatraemia in this patient could only be explained by an inappropriately high circulating concentration of antidiuretic hormone.

We do not know why so much antidiuretic hormone was released in this patient. Release of the hormone is controlled not only by local osmotic factors but also by neurogenic stimuli via the vagus and glossopharyngeal nerves, initiated by the effects of changes in systemic haemodynamic function on baroreceptors in the aortic arch and carotid sinus. ${ }^{67}$ The vagus seems to inhibit secretion of antidiuretic hormone. ${ }^{8}$ Alpha and beta sympathetic stimulation have opposing effects on the parasympathetic tone within these pathways and so also contribute to the control of the release of antidiuretic hormone. ${ }^{7}$ These nerve pathways, however, were unlikely to have been affected by the spinal injury in this patient so they were probably not part of the underlying pathological mechanism. An alternative hypothesis is that pericardial fluid reduced local afferent vagal tone from the pericardium and so lessened the inhibition of the release of antidiuretic hormone. Induction of cardiac tamponade in anaesthetised dogs was associated with decreased afferent nerve traffic from the pericardium via the right cardiac nerve. ${ }^{9}$ This local factor could exaggerate the usual neurogenic stimuli for release of antidiuretic hormone, which are a consequence of the haemodynamic effects of cardiac tamponade.

We conclude that hyponatraemia may develop when there is an imbalance between the antinatriuretic and antidiuretic responses to cardiac tamponade and that it will resolve once the tamponade is relieved.

We thank Dr R J C Hall for his advice, comments, and permission to report this case.

1 Raff H, Cogswell TL, Bernath GA, Klopfenstein HS. Vasopressin and ACTH responses to acute cardiac tamponade in conscious dogs. [Abstract] Clin Res 1986;34:899A.

2 Mancini GBJ, McGillem MJ, Bates ER, Weder AB, DeBoe $S F$, Grekin RJ. Hormonal responses to cardiac tamponade: inhibition of release of atrial natriuretic factor despite elevation of atrial pressures. Circulation 1987;76:884-90.

3 Edwards BS, Zimmerman RS, Schwab TR, Heublein DM, Burnett JC Jr. Atrial stretch, not pressure, is the principal determinant controlling the acute release of atrial natriuretic factor. Circ Res 1988;62:191-5.

4 Koller PT, Grekin RJ, Nicklas JM. Paradoxical response of plasma atrial natriuretic hormone to pericardiocentesis in plasma atrial natriuretic hormone to pericardiocent

5 Osborn JL, Lawton MT. Neurogenic antinatriuresis during development of acute cardiac tamponade. Am J Physiol development of acute

6 Baylis PH. Osmoregulation and control of vasopressin secretion in healthy humans. Am J Physiol 1987; 253:R671-8.

7 Schrier RW, Berl T, Harbottle JA, McDonald KM. Catecholamines and renal water excretion. Nephron 1975;15:186-96.

8 Schrier RW, Berl T. Mechanism of the antidiuretic effect associated with interruption of parasympathetic pathways. J Clin Invest 1972;51:2613-20

9 Castaner A, Kostreva DR, Kampine JP. Changes in autonomic nerve activity during acute pericardial tamponade. Cardiology 1980;66:163-73. 